\title{
Sexual snakes, winged maidens and sky gods de Serge Dunis (éd.)
}

\section{Raymond MAYER}

\section{(2) OpenEdition \\ Journals}

Édition électronique

URL : http://journals.openedition.org/jso/6331

DOI : $10.4000 /$ jso.6331

ISSN : $1760-7256$

Éditeur

Société des océanistes

Édition imprimée

Date de publication : 30 juin 2011

Pagination : 197-199

ISBN : 978-2-85430-030-7

ISSN : 0300-953x

Référence électronique

Raymond MAYER, "Sexual snakes, winged maidens and sky gods de Serge Dunis (éd.) », Journal de la Société des Océanistes [En ligne], 132 | 1er semestre 2011, mis en ligne le 30 juin 2011, consulté le 21 septembre 2020. URL : http://journals.openedition.org/jso/6331 ; DOI : https://doi.org/10.4000/jso. 6331 
pour l'Océanie et ses «Naturvölker » dont ce livre porte témoignage (jusqu'à utiliser cette vieille dénomination). Mais il a estimé ces sentiments ou ces expériences suffisamment familiers à ses lecteurs pour s'autoriser à leur présenter un tableau composite de la Mélanésie, mêlant les observations récentes consignées à la faveur de ses voyages et les documents remontant à l'époque coloniale, quitte à donner l'impression que, pour diverses îles et leurs populations, l'histoire s'est arrêtée en 1914, ou au départ subséquent des Allemands.

Ce tableau ordonné géographiquement s'intéresse d'abord à divers groupes de Nouvelle-Guinée puis à certains points de la Mélanésie insulaire, sans souci d'exhaustivité ni de justification du parcours adopté. Des exposés livresques qu'il offre des Trobriand, de l'archipel Bismarck et des îles Salomon du nord-est, de Santa Cruz, de la Nouvelle-Calédonie et de l'archipel de l'Amirauté, les spécialistes retiendront surtout l'iconographie, reproduisant souvent des documents peu connus issus de musées allemands avec lesquels R. Garve collabore depuis longtemps, ceux de Dresde et de Leipzig en premier lieu. Mais les observations recueillies au Vanuatu entre 1994 et 2009, notamment à propos de la révolte de Jimmy Stevens et des survivances du culte du cargo (pp. 172-179), méritent certainement d'être lues avec l'attention qu'on réserve d'habitude aux rapports ethnographiques.

C'est pour la Nouvelle-Guinée, que R. Garve a le plus parcourue et où $\mathrm{M}$. Garve l'accompagne depuis une décennie, que ce volume livre le plus grand nombre d'informations de première main. A côté des généralités concernant les Marindanim, les Abelam ou les habitants des rives du Sepik (ceux du golfe Papou sont parmi les absents notables de ce livre), toute considération est due aux chapitres qu'il consacre aux Korowai (visités en 1999, 2002, 2004), aux Asmat (visités en 1986 et 1988), aux Dani $(1986,1988,1989,2002$, 2003), aux Yali (1988, 1989, 2002), aux Mek (1989 puis 2004 et 2006), aux nomades des marais du Memberamo (2007), et aux Din, " groupe jusque-là inconnu d'Irian Jaya [actuelle Papouasie occidentale] », dont l'accès avait été facilité aux explorateurs par leurs voisins Korowai en 2002. Ces textes dépassent largement en intérêt les titres parfois complaisants dont ils sont pourvus, tels "les Mek, sans doute les plus petits des humains", " les Yali, guerriers aux ceintures de rotin" ou «les Dani, étuis péniens et haches de pierre ». Du reste, dans ce dernier exemple, il décrit largement plus les procédés de production du sel des Dani que leurs étuis péniens (holim ou koteka pp. 67 et 72), signalant surtout la récente interdiction de leur port dans les Hautes Terres, le Parlement indonésien les ayant jugés "pornographiques ».

Ces pages offrent maintes autres notations tirées de l'histoire récente ou de la pratique médicale et scientifique des deux auteurs, qui ont tenu à compléter leur ouvrage par un dévelop- pement sur les principales plantes alimentaires (pp. 218-221) complété par un «petit $A B C$ » des plantes médicinales et condimentaires de Mélanésie (pp. 228-235), ainsi que par un exposé sur "l'histoire du peuplement humain de l'Océanie d'après les découvertes génétiques" (pp. 222227) dû à Manfred Kayser, titulaire de la chaire de biologie à l'université Erasmus de Rotterdam. Il y résume les recherches qu'il a menées avec d'autres savants sur la transmission de ces marqueurs que sont le chromosome $\mathrm{Y}$ et les mitochondries au sein des populations océaniennes, suggérant d'intéressantes hypothèses sur les sociétés austronésiennes matrilinéaires et matrilocales disposées à accueillir des hommes d'origine papoue, tandis que les sociétés papoues, d'abord polygynes, patrilinéaires et patrilocales, auraient tendu à envoyer au loin leurs jeunes gens surnuméraires ou contraints au célibat.

Pour les lecteurs qui verront dans ce volume surtout un recueil d'images spectaculaires ou peu connues, ce qui constitue en effet une de ses qualités, il faut signaler que soixante-deux des photos reproduites en pleine page sont dépourvues de légende et imposent d'aller chercher des éléments d'information dans les crédits photographiques ou le texte courant, qui les fournit la plupart du temps. Pour ceux que l'ouvrage intéressera pour ses observations inédites, ou comme initiation à certaines questions scientifiques posées aux Océanistes d'aujourd'hui, on recommandera de se reporter à d'autres ouvrages plus développés de R. Garve, tel celui qu'il a publié avec Frank Nordhausen, Laleo. Die geraubte Steinzeit ( L'âge de pierre volé ", Christoph Links Verlag, Berlin, 2009), où il consacre par exemple aux Korowai (pp. 74-102) un exposé nettement plus fourni que dans ce volume-ci (pp. 30-43), dont les informations concises ne sont déjà pas à négliger.

\section{Gilles BounOure}

Dunis Serge (éd.), 2008. Sexual snakes, winged maidens and sky gods, Nouméa - Pape'ete, Le Rocher-à-la-voile - Haere Po, 286 p., bibliogr., dessin au trait et carte.

Dans un format in-octavo propice à la circonstance, $S$. Dunis a su célébrer le centième anniversaire d'un Claude Lévi-Strauss alors vivant, en publiant en anglais, dans une opération conjointe des éditions Le Rocher-à-la-Voile (Nouméa) et Haere Po (Pape’ete), un bel ouvrage de 287 pages qui réunit une pléiade d'auteurs confirmés ou en voie de l'être, sur des thèmes de mythologie offerts en bouquet numérique (puisque le livre est aussi édité en version électronique) à l'auteur des «Mythologiques».

On connaissait le serpent à plumes des Incas, le lion ailé de Venise ou les dieux d'eau d'Ogotemmèli. Voici que le livre imaginé et coordonné par Serge Dunis nous entraine vers des serpents 
sexuels, des jeunes filles volantes et des dieux célestes! L'ouvrage, dont la genèse remonte à 2002, est devenu un cadeau dédié au centenaire de Claude Lévi-Strauss, et un dessin au trait, portraiturant le dédicataire au revers de la page de garde, signe d'ailleurs le livre. Un long article de Jean Guiart le préface, et un texte de Serge Dunis revendiquant une alliance préférentielle entre la physique nucléaire et la mythologie le conclut. Le ton est déjà donné. Faire admettre l'étude des mythes comme contribution scientifique à part entière à l'intelligence des processus historiques de la circulation de la parole et des imaginaires n'est pas une mince affaire, et cela explique le climat suspicieux qui entoure généralement ce type d'entreprise.

Comme il fallait s'y attendre - et à l'instar de son aîné Stéphane Hessel -, Jean Guiart a la faculté d'indignation intacte. Dans sa préface de quarante pages, il va méthodiquement l'appliquer à tous les sujets qui semblent aller de soi et à tous les auteurs qui, selon lui, font fausse route. La première salve d'indignation est amicalement dirigée en direction de Marshall Sahlins (pp. 1112). Jean Guiart lui fait grief d'avoir commis la même erreur que James Cook à Aoba, en interprétant aux Fidji certains cas de décoloration dermique comme de la lèpre. Il conteste la mise à mort d'une femme lépreuse, de même que l'administration de poison à un nouveau chef aux Lau (alors qu'il s'agit simplement de kava vert). Il revient aussi sur la célèbre assimilation sahlinsienne du capitaine Cook au dieu Lono, en lui opposant le double argument de la menace militaire que représentaient les Blancs - menace très tôt reconnue par les Océaniens à partir de rencontres antérieures de Japonais et de Russes - et de l'absence de toute divinisation des Blancs chez les Hawai'iens, tout comme chez les Tongiens quelque temps plus tôt, ou chez les Maori quelque temps plus tard, les uns et les autres cherchant systématiquement à s'emparer des armements des Occidentaux.

La deuxième bordée d'indignation (p. 12) s'adresse aux linguistes qui ne reconnaissent pas certaines caractéristiques (dont les langues à tons) des langues austronésiennes ou qui opposent les "langues dites papoues" aux langues austronésiennes sans avoir préalablement achevé de décrypter l'extrême diversité des premières. Une troisième volée de bois vert est administrée aux chercheurs faisant mauvais usage de la notion de " mythe » à partir de critères ethnocentriques ou de conceptions généralisées sans nuances. Le qua trième tir de barrage vise la qualité des collectes de "mythes" antérieures à 1925 . Ne trouvent grâce à ses yeux que les travaux de collecte réalisés par les Allemands Karl von den Steinen aux Marquises et Augustin Kraemer aux Samoa, du fait de leur collecte systématique des éléments de contexte et de notes substantielles accompagnant les textes. Les travaux de Teuira Henry sont dis- qualifiés (p. 13), parce que tenus pour des traductions « romantiques » ignorantes de la toponymie stratégique investie dans les textes essentiels.

Viennent les quatre règles d'or applicables à toute étude de mythe: 1 . Collecte directe sur le terrain dans les termes de leur énonciation originale ; 2 . Collecte de tous les détails sans interrompre l'énonciateur; 3 . Collecte des faits liés à la compréhension du récit; 4 . Collecte non d'un récit unique mais de toutes ses variantes. À ces règles élémentaires s'ajoutent toutes les qualités de mobilité et de durée nécessaires à une ethnographie méticuleuse, attentive aux dispositifs de tenure foncière et capable d'apprendre à travers mille et un détails imprévus plutôt qu’à partir de positions dogmatiques statiques.

La préface touche plus d'une vingtaine de thèmes que Jean Guiart avait manifestement à cœur de récapituler en langue anglaise. Ils vont du "maittre de Koné » jusqu'aux mouvements millénaristes dont il rappelle l'intervention personnelle déployée pour faire adopter des positions de tolérance de la part des administrations britanniques et françaises dans les Nouvelles-Hébrides de l'époque. Quel que soit le ton employé, nous voici à tout le moins renseignés sur ce qu'il convient de réformer dans nos entendements scientifiques tant soit peu figés et insuffisamment ouverts aux thèses innovantes!

Comme le reconnaît Serge Dunis lui-même (p. 8), la préface de Jean Guiart est un livre dans le livre, et certaines de ses positions contreviennent sans doute à ses termes de référence (l'affichage de divinités célestes par exemple) mais chacun trouve avantage à communiquer ses thèses maîtresses en langue anglaise, tout en admettant un certain pluralisme dans les positionnements scientifiques défendus par les uns et les autres. L'ouvrage vaut non seulement par ses thèses générales mais aussi par celles introduites par les chapitres particuliers.

Les douze chapitres de l'ouvrage sont annoncés (plutôt deux fois qu'une, pp. 51-65) par son éditeur scientifique, qui a déjà présenté dans un propos liminaire la "généalogie " de l'ouvrage. Celui-ci a été progressivement développé à partir de symposiums jusqu'en réseaux électroniques, et de relectures jusqu'en adjonctions s'étalant tout au long de la période 2002-2008, rappelant au passage le black-out de 2007 dû à une thérapie sévère d'un cancer heureusement surmonté.

Les chapitres ne reprennent pas les trois figures emblématiques du titre (serpents, jeunes filles et dieux) mais sont distribués en fonction d'une thématique générale où les couples eros/thanatos et pouvoir/parité occuperaient les sommets, et les articles les intervalles. La matière est ainsi répartie entre une variété de thèmes intitulés "La mer", "L'espace et le temps infinis", "Le sexe cru», " Inceste et pouvoir politique ", "Une femme initiée en vaut deux ", "Sexe sublimé et libre sexe ", 
"Super-femme mélanésienne et beauté polynésienne ", "Création et suprématie masculines". On pressent, à travers cet étiquetage, que le parcours est dédié au moins autant à la psychanalyse qu'à l'anthropologie.

Échappant quelque peu à cet environnement psychanalytique, Ben Finney revient (pp. 69-79) sur les motivations des navigations transpacifiques qu'il a ressuscitées à partir de Hawaii en 1976. Il en signale les inspirations "mythologiques".

Le plan de l'ouvrage obéit ensuite aux nécessités d'une large couverture du domaine géographique, en autorisant chaque auteur à pratiquer un commentaire approprié sur le mythe (ou la série de mythes) qu'il a privilégié sur son terrain propre. Doug Dalton (pp. 83-98), de l'université Longwood, procède à une analyse structurale du récit de source rawa (Papouasie NouvelleGuinée) sur les "larmes des étoiles". Ce récit explique l'origine de la pluie comme le résultat mythique de l'échec des étoiles à pouvoir compter les grains de sable, dans le défi réciproque que les étoiles et le sable s'étaient lancé.

Jukka Siikala, de l'université d'Helsinki (pp. 99109), procède de même sur un récit généalogique sur les origines de Tangaroa aux îles Cook pour reconstruire l'univers à partir d'une chaîne de dualités qui se répètent tout en se différenciant.

Roger Lohmann (pp. 113-125), de l'université Trent, pratique le même exercice sur le mythe asabano (Papouasie Nouvelle-Guinée) du vieil homme figure de l'espiègle et du héros civilisateur, pour en obtenir une expression de l'immortalité. C'est dans son explication que les serpents sont doublement référés à la sexualité phallique et aux mues de l'immortalité.

Serge Dunis (pp. 129-140) revient aux études qu'il a menées sur le mythe de création maori découlant de l'inceste des jumeaux Rangi (ciel) et Papa (terre), pour en déduire des résultats qu'il arrive à rendre congruents avec les mythes de la patate douce, la hiérarchie hawaïenne et le triangle polynésien.

Marika Moisseeff (pp. 141-154), du CNRs à Paris, conduit son analyse jusqu'à conjoindre la mythologie polynésienne de "l'île des femmes " sans hommes, avec des épisodes de la série américaine Alien, en les constituant en autant de répliques de l'allégorie trouble de la reproduction/destruction.

Unasa Leulu Felise Vảa (pp. 157-172), de l'université nationale de Samoa, nous gratifie d'explications limpides sur un récit samoan de l'héroïne Sina et de son frère Lupe (pigeon) qui remonte à la période du premier converti à la London Missionary Society.

Solange P. Skinner, antérieurement de la mission scientifique française à Fidji (pp. 173-180), prend à témoins les mythes d'une île (Nauru) matrilinéaire où les femmes sont censées régir la terre et les hommes régner sur les océans, pour montrer que l'image masculine n'est en rien minorée par des principes de prééminence féminine.
Naomi M. McPherson (pp. 183-201), du collège universitaire Okanagan, exerce sa méthode sur des mythes d'origine de la crécelle, du taro et du sagou dans la culture bariai de Nouvelle-Bretagne pour conclure que les dualismes cartésiens sont inadéquats pour faire comprendre des rapports complexes de genre et de sexualité.

Heather Young Leslie (pp. 203-224), de l'université de Hawai' $i$, prend appui sur un récit de pêche tongien pour décrire le processus de légitimation d'un chef à travers diverses configurations incarnées par la faune marine.

Wolfgang Kempf (pp. 227-238), de l'université de Göttingen, nous installe chez les Ngaing de Papouasie Nouvelle-Guinée pour y connecter les pratiques rituelles au contexte social à la la faveur d'un récit dialogué.

Jean-Guy Cintas (pp. 239-250) enfin, de l'université de Bordeaux, complète la série des analyses du livre par celle d'un corpus de chants généalogiques du Kumulipo de Hawai'i et s'interroge sur les différences de traitement d'un mythe et d'un poème.

Dans chaque chapitre, le lecteur est appelé à partir à la rencontre d'un ou plusieurs textes de référence, qui sont, à leur tour, soumis à une ou plusieurs analyses dont la méthode revendique, peu ou prou, une approche de type lévi-straussien, mais qui répond de fait à l'expérience acquise par chaque auteur sur son terrain privilégié. Dans leur ensemble, les chapitres poursuivent une réflexion multidisciplinaire où les concepts psychanalytiques rivalisent parfois avec ceux de l'anthropologie - perspective que récuse d'ailleurs explicitement le scrutateur final, Ward H. Goodenough (pp. 253-258), professeur émérite de l'université de Pennsylvanie, qui lui aussi en profite pour retourner à ses thèmes de prédilection (relations entre germains de même genre ou de genre opposé, cosmogonies des $\mathrm{Ca}$ rolines et aspects légendaires des mythes).

Serge Dunis (pp. 259-267) se réserve l'exposition finale en connectant la mythologie à la physique nucléaire. Même s'il avoue que son propos est purement métaphorique, sa proposition de fragmentation atomique en dix-huit hypersymétries $(s i c)$ irradiant les thématiques mythiques océaniennes n'entraînera certainement pas l'adhésion spontanée de ses lecteurs. On conviendra que le style de l'ouvrage est volontiers épique et entend probablement toucher un public plus large que le public scientifique standard. Mais la déconstruction, tant des méthodes d'interprétation que des énoncés auxquels elles s'appliquent, suscite un intérêt certain. De ce point de vue, l'ouvrage pourrait être tenu pour un cadeau fait aux dieux et aux hommes, qu'ils aient des ailes ou non!

Raymond MaYer, Univ. Omar Bongo Libreville et Lumière-Lyon2 